OPEN ACCESS

Edited by:

Pei Zhang,

Xi'an Jiaotong University, China

Reviewed by:

Dong Mao,

Northwestern Polytechnical

University, China

Cuicui Lu,

Beijing Institute of Technology, China

*Correspondence:

Shlomi Lightman

shlomili@soreq.gov.il

Specialty section:

This article was submitted to

Optics and Photonics,

a section of the journal

Frontiers in Physics

Received: 14 December 2021

Accepted: 17 January 2022

Published: 04 March 2022

Citation:

Lightman S, Wengrowitcz JM, Teitelboim A, Gvishi $R$ and Hurvitz $G$ (2022) Exploring for New Insights in the Performance of a 3D Orbital Angular

Momentum Mode-Sorter.

Front. Phys. 10:835159.

doi: 10.3389/fphy.2022.835159

\section{Exploring for New Insights in the Performance of a 3D Orbital Angular Momentum Mode-Sorter}

\author{
Shlomi Lightman ${ }^{1 *}$, Jonathan M. Wengrowitcz ${ }^{2}$, Ayelet Teitelboim ${ }^{1}$, Raz Gvishi $^{1}$ and \\ Gilad Hurvitz ${ }^{1}$
}

${ }^{1}$ Applied Physics Division, Soreq NRC, Yavne, Israel, ${ }^{2}$ Physics Department, Weizmann Institute, Rehovot, Israel

Optical vortex beams are light beams that can carry orbital angular momentum (OAM). Hence, such beams may serve as potential candidates for carriers of information in optical communication and quantum optics applications. This is owing to their spatial orthogonality, as these beams can be combined (multiplex) or separated (demultiplexed). We recently demonstrated a new method to detect OAM states by using a 3D-direct laser printing fabrication process. Measuring the mode-sorter performance was challenging, mainly due to mechanical and optical sensitivities originated from misalignments. In this work, this sensitivity was thoroughly examined. Pure OAM states having lateral and angular misalignments relative to the mode-sorter were introduced, and cross-talk between resolved states was theoretically simulated. The system is relatively vulnerable to small misalignments, which challenge its implementations in free-space communication systems. However, this might be an advantage for counseled communication, in which eavesdropping becomes more challenging, due to the angle-dependent increased modal cross-talk.

Keywords: vortex beam, 3D printing, orbital angular moment, mode sorter, phase modulated

\section{INTRODUCTION}

Optical vortex beams are characterized by a winding azimuthal phase, exp (il $\phi)$, where $l$ is the topological charge and $\phi$ the azimuthal angle [2]. These beams can carry orbital angular momentum (OAM). This unique property enables implementations of such beams in various applications, including particle manipulation [3], microfabrication [4], astronomy [5], quantum optics [6], highresolution microscopy [7], and space-division multiplexed communication systems [8]. There are several types of beams that can carry OAM. A commonly used type is the Laguerre-Gaussian (LG) family of beams which is an example of a set of vortex beams that are also solutions of the paraxial Helmholtz equation. In this case, beams with different OAM values are orthogonal; hence, they can be spatially multiplexed and demultiplexed on the same physical channel. These beams can then be used as carriers of information, thereby increasing the information capacity of an optical communication channel [9]. An efficient way to separate OAM states is by applying a Cartesian to log-polar transformation, based on two refractive optical elements [10]. This transformation maps the azimuthal phase profile of an OAM mode into a tilted planar wavefront. As a result, linear combination of OAM states can be simultaneously transformed into a set of planar waves with tilted wavefronts, where the tilt angle depends on the topological charge. These tilted waves can therefore be easily separated in the far field by a lens, enabling OAM analysis, as each mode is located at a different location on a detector. The desired two refractive optical elements are characterized by a 
non-trivial 3D shapes. Hence, reaching small scale, 3D, and high optical quality is a challenging task. Conventional implementations of such transformation are based on spatial light modulators (SLMs) [11], or large-scale (cm) diamondturned surfaces [10]. In general, fabrication or manufacturing of small-scale (below hundreds of micrometers) high-quality optical surfaces, characterized by tens of nanometer surface roughness (RMS) is a challenging task. An innovative new approach was able to minimize the two elements into only one, along with $2 \mathrm{D}$ high-resolution diffractive surfaces using electron beam lithography $[12,13]$. Furthermore, the generation of vortex beams $(l \leq 1)$ and mode analysis was demonstrated in an all-fiber device [14]. However, all mentioned approaches do not provide direct integration to other optical elements (such as optical fiber), enable costeffective processes, or provide simple and straightforward lithography procedures for various states. Our recent work showed a new fabrication method that provides high-quality and integrated mode-sorter, based on the two optical elements [1]. This method used a 3D-Direct laser writing (3D-DLW) [15] system, which is based on a nonlinear two-photon absorption process [16]. This concept allows reaching accurate 3D elements of complicated surfaces using the polymerization of a lightsensitive material volume (Voxel). Although implementations of 3D-DLW technology for micro-optics devices are widely reported [15-20], we were able to harness, for the first time, this platform for analyzing OAM modes using the transformation optics theory [21, 22]. This approach may induce new and exciting opportunities for vortex beam detection and analysis in integrated devices, in communication systems, or for quantum optical applications. In our previous work [1], we demonstrated that the two-element $3 \mathrm{D}$-printed free-space mode sorter is capable of handling both pure and mixed vortex beams with topological charges $l \leq|3|$. These results were rather preliminary, as further analysis was needed to explore its functionality and performance. Some of the remaining open questions regard the system capabilities upon lateral misalignment and optical directionality (i.e. incoming tilted waves). Hence, in this work, these issues are examined, as various calculations were performed to study the sensitivity of this system.

\section{PRINCIPLE OF THE OAM MODE-SORTER}

The Cartesian to log-polar transformation is constructed by two refractive optical elements [11]. These two elements map the coordinates of the incoming beam (in the $x-y$ plane) to an output plane $(u-v)$ using the following relations:

$$
\begin{gathered}
u=-a \cdot \ln \left(\frac{\sqrt{x^{2}+y^{2}}}{b}\right) \\
v=a \cdot \tan ^{-1}\left(\frac{y}{x}\right)
\end{gathered}
$$

The coordinates $u$ and $v$ describe the Cartesian coordinates at the first element's Fourier plane. The parameter $a$ is a scaling factor, equals to $d / 2 \pi$, as $d$ is the width of the second element, and

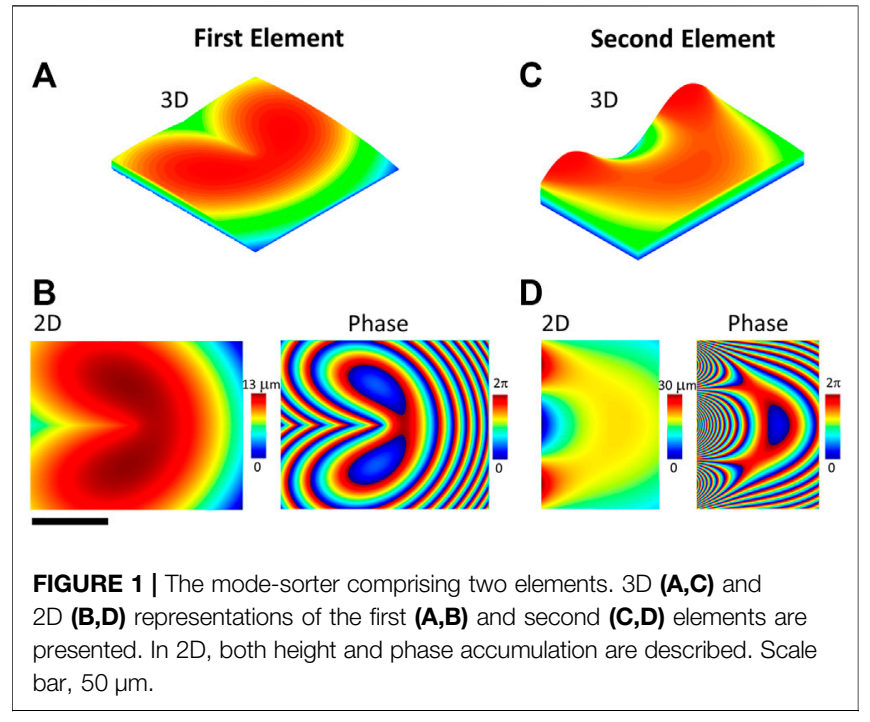

$b$ determines the beam location along the $v$ axis. The elements' surfaces are hereby described:

$$
\begin{aligned}
Z_{1}(x, y)=- & \left(\frac{a}{f\left(n_{m}-n_{\text {air }}\right)}\right)\left[y \cdot \tan ^{-1}(y / x)-x \cdot \ln \left(\frac{\sqrt{x^{2}+y^{2}}}{b}\right)\right. \\
& \left.+x-\frac{1}{2 a}\left(x^{2}+y^{2}\right)\right] \\
Z_{2}(u, v)= & -\left(\frac{a \cdot b}{f\left(n_{m}-n_{\text {air }}\right)}\right)[\cos (v / a) \cdot \exp (u / a) \\
& \left.-\frac{1}{2 a \cdot b}\left(u^{2}+v^{2}\right)\right]
\end{aligned}
$$

$Z_{1}$ and $Z_{2}$ describe the height profiles of the first and second element, accordingly. The distance between the elements is determined by $f$, and the material and air refractive indices are denoted as $n_{m}$ and $n_{\text {air }}$ accordingly. Modes carrying OAM values that pass through the second element are characterized by rectangular shapes and tilted wave-fronts, dictated by $2 \pi l$. Introducing a lens to the tilted waves will result in a focus movement perpendicular to the elongated diffraction limited spot size, by the following connection:

$$
\Delta=l \cdot\left(\frac{\lambda f}{d}\right)
$$

\section{THEORETICAL DESIGN AND CALCULATIONS}

In order to reach optimal phase elements, based on the two surfaces provided in Eqs 3, 4, a simulation code based on a splitstep Fourier method [23] was used to study the OAM beam propagation through those surfaces. In this way, the elements' functionality was explored and tailored to the experimental system, as the free parameters were determined. The 


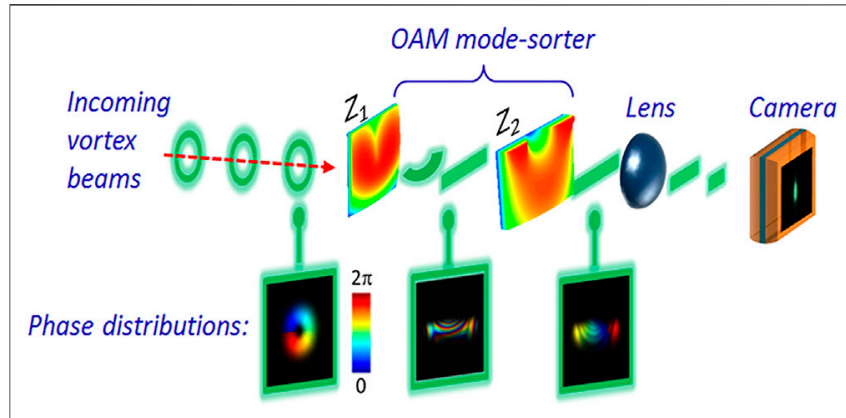

FIGURE 2 | Schematic illustration of the mode-sorter as incoming / = 1 OAM mode propagates through the elements. Additional phase and intensity profiles are presented at certain locations with respect to the two elements.

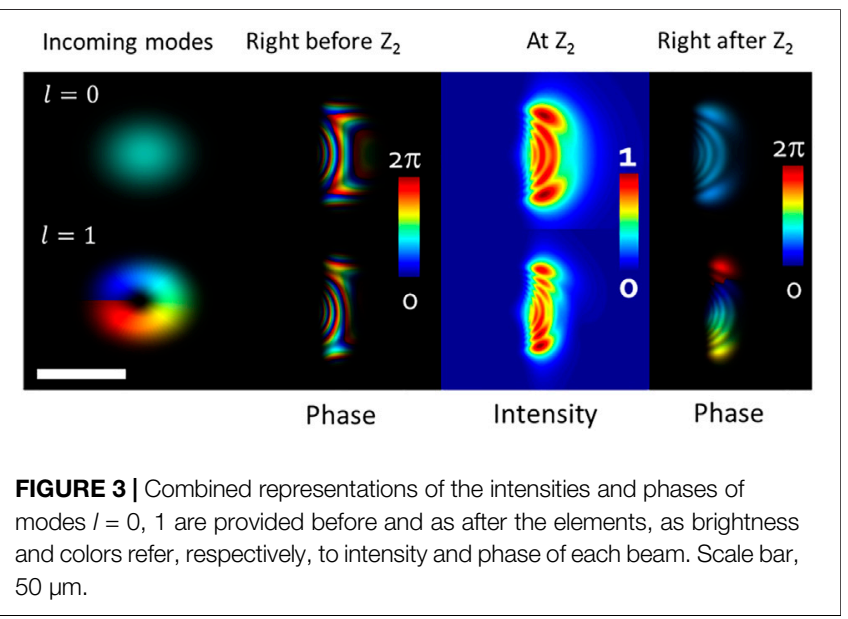

parameters $a, b$, and $f$ were chosen to be 19,25 , and $800 \mu \mathrm{m}$, accordingly. The final obtained elements are brought in Figure 1, as $3 \mathrm{D}$ structural representation (top) and $2 \mathrm{D}$ surfaces and phase modulations (bottom) can be seen. The lateral dimensions of the first element were chosen to be $100 \times 100 \mu \mathrm{m}^{2}$ reaching $12.5 \mu \mathrm{m}$ at maximum height. The second element lateral size was $160 \times$ $100 \mu \mathrm{m}^{2}$ with maximal height of $30 \mu \mathrm{m}$. Figure 2 provides a schematic illustration of the operating principle. Here, an OAM mode of $l=1$, characterized by a ring-shaped intensity profile passes through the first element, as the ring gradually becomes open until reaching a rectangular shape at the second element plane. The second element return the mode's initial phase. Then, a lens focuses the mode into an elongated shape at the detector. Combined representations of the mode's intensity and phase are also provided before and after the elements, as brightness and colors refer, respectively, to intensity and phase of each beam. Figure 3 provides further understanding of the OAM mode sorter operation, as front view intensity and phase calculations of $l=0$ and $l=1$ modes can be seen in the vicinity of Element 2 (surface $\mathrm{Z}_{2}$ ). Here, the two modes possess unresolved phase distribution prior entering the element. However, passing through the second element reorganizes the phases according to the OAM values. It is worth emphasizing that the intensity profile of all modes remains the same before and after each element, as only the phase is

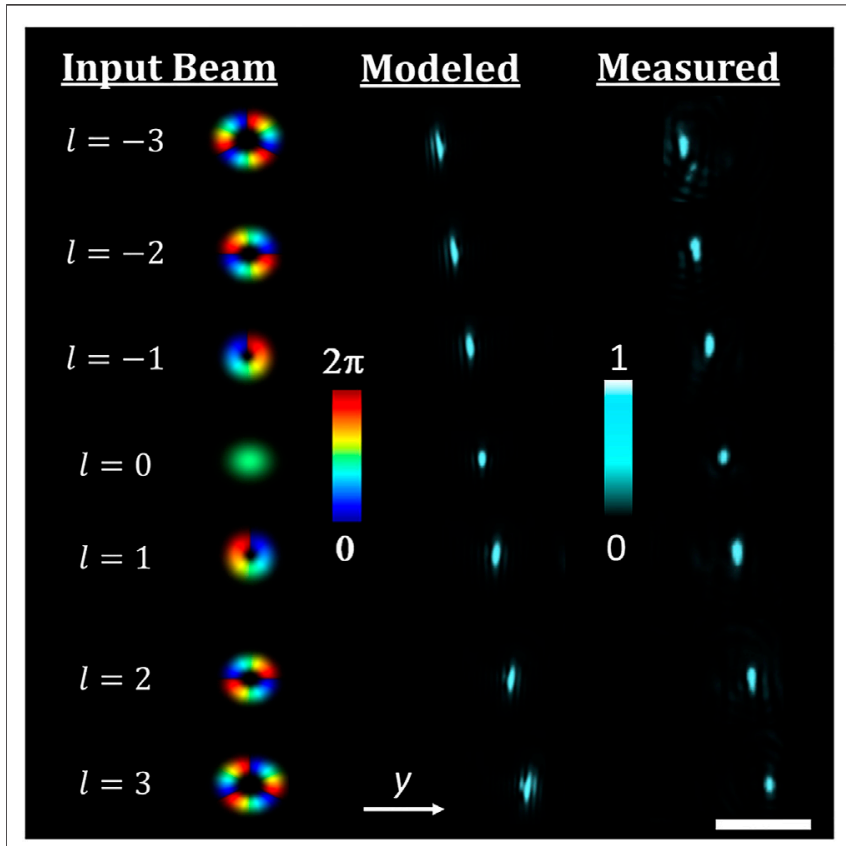

FIGURE 4 | Simulation results of a free-space OAM mode-sorter followed by experimental results. Each OAM state propagates onto a different location on the detector, with correlation to the state's topological charge. Scale bar, $100 \mu \mathrm{m}$.

transformed, which enables a gradient phase change that is manifested in the focused spot position variations on the detector.

\section{NOMINAL PERFORMANCE OF THE MODE-SORTER}

The nominal performance of the OAM mode-sorter is hereby described. This means that OAM states were injected to the modesorter perpendicular to the elements, with no tilt or spherical aberration. The detector that registered the incoming modedependent spots was also located at the center of the beam's axis (for $l=0$ ). This provides a reference point for further understanding the sensitivity and misalignment issues. By using a measurement system, consisting of a CW laser source $(690 \mathrm{~nm})$, phase-only spatial light modulator (SLM), and additional optics, the mode-sorter performance was examined, according to a previous work [1]. The SLM provided various OAM modes, $-3 \leq l \leq 3$, that propagated toward the mode sorter two-element system, which were than detected using a CCD. The element fabrication process, detailed measurement system, and additional results and information are further elaborated in a previous work [1]. Figure 4 provides the experimental results along with predicted simulations of the modesorter functionality for the mentioned OAM values at the detector plane. The incoming modes are also provided for convenience. It can be seen that the modes are clearly resolved, as various states are introduced to the system. High $l$ values may cause fringes that lower the ability to determine the OAM state. In general, small beams have short Reighley length. When working with longer distances, the beam 


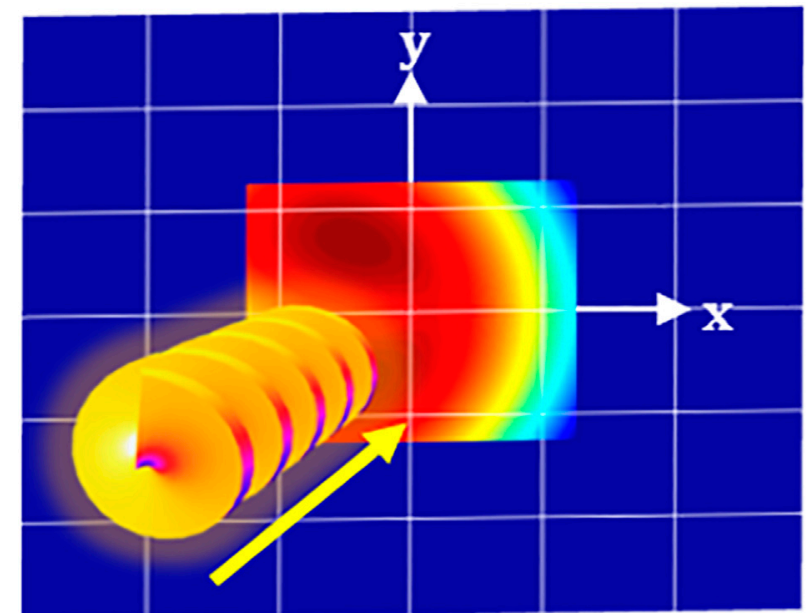

FIGURE 5 | Schematic representation of the lateral displacement calculative method. The entire two-element system moves together in each direction (only the first element is shown). carries a spherical phase front rather a plane one. This induces fringes and reduces the device performance to detect accurately OAM states. Furthermore, the influence of production inaccuracies tends to minimize as dimensions increase. The mode sorter consists of two refractive phase elements. The coupling loss for an incoming $\mathrm{LG}_{10}$ mode in this system is $30 \%$. The main cause for such losses is due to Fresnel reflections. Additional losses such as absorption and diffraction caused by the device itself are negligible. This is because it is a phase-only device that is made of a transparent polymer.

\section{SORTING SENSITIVITY}

In this chapter, the system performance is investigated under lateral misalignment of the incoming beam. Such misalignment often happens in free optic applications. This was carried out by moving the mode-sorter laterally to the beam propagation axis. Since the twophase structures consisting the mode-sorter device have a complex 3D surface, the performance and OAM spectral purity are expected to be affected. It is convenient to describe the OAM of an LG light beam by its optical axis. Hence, by choosing different axis, these light beams could be represented by a span of various LG modes, which also serve as orthogonal states of the paraxial wave equation, providing discrete OAM spectrum. A lateral displacement of a symmetric beam will not alter its OAM value [24]. However, such a displacement may alter a single photon OAM state, with respect to the reference axis. Meaning, the pure OAM state of an individual photon will be represented by a superposition of many OAM states in the new displaced coordinate system [25]. In other words, by changing the observation axis, the mode purity is disturbed and redistributed as a span of discrete states. Hence, the ability to analyze the OAM state of a certain mode becomes more challenging due to this spectrum broadening. As shown here and in [1], the mode-sorter is capable of detecting various OAM states. However, exploring the system's performance upon displacements or due to beam wondering effects should be further investigated. In this
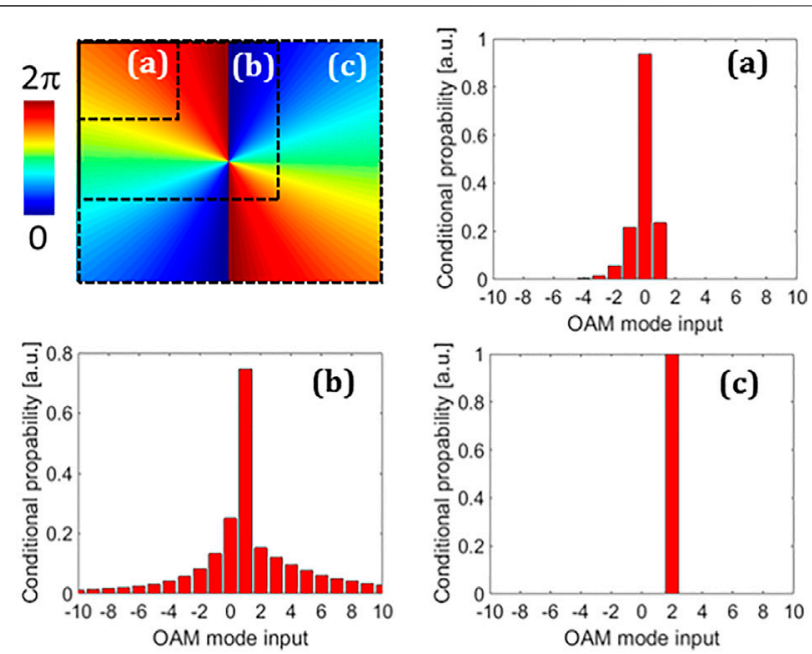

FIGURE 6 | OAM attained spectrum, in the case of $I=2$, when phase analysis is carried out for different areas and locations in the phase distribution. The pure state is screened, as modal spanning is formed.

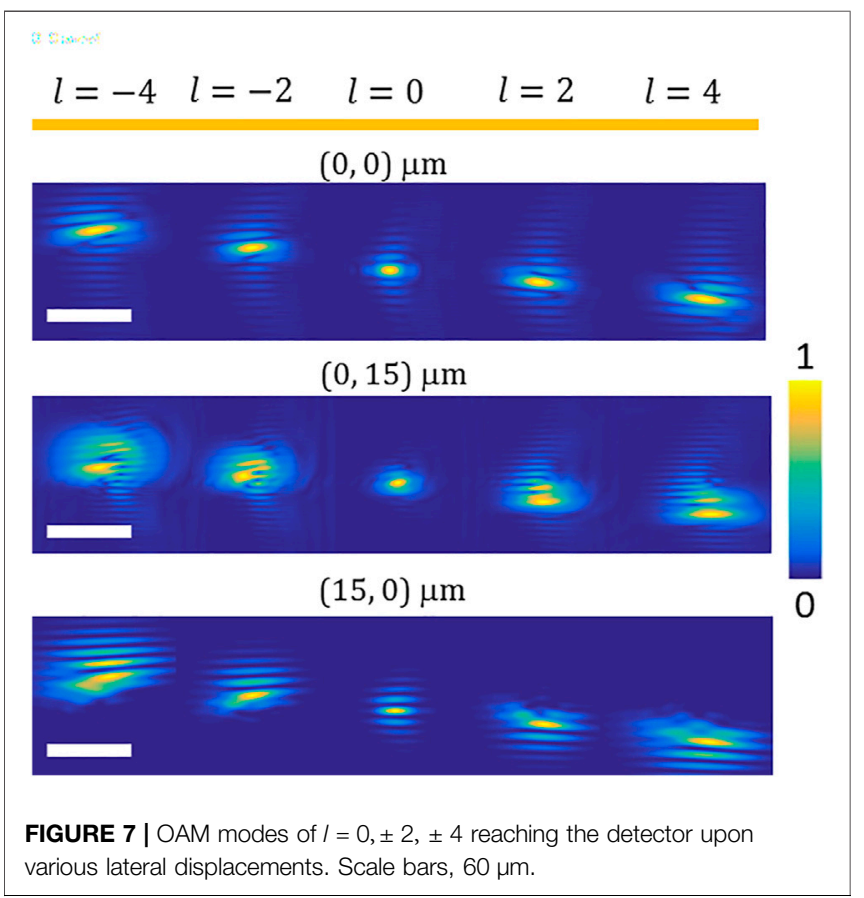

work, we characterize theoretically the system ability to detect incoming OAM states, when the whole mode-sorter system is displaced from its center $(x=0, y=0)$, and also when the modesorter is not displaced but is introduced to tilted modes at different angles. Figure 5 provides a schematic illustration of the calculation principle. The mode-sorter, as one system, is displaced to various $(x, y)$ locations, with respect to the axis origin, and then introduced to incoming LG modes carrying different OAM values. As a first step, we show an example that exhibits the challenging task to detect the OAM spectrum solely from the incoming spiraling phase front. This is given in Figure 6, where the phase of OAM state $l=2$ (Figure 6, top left) is 


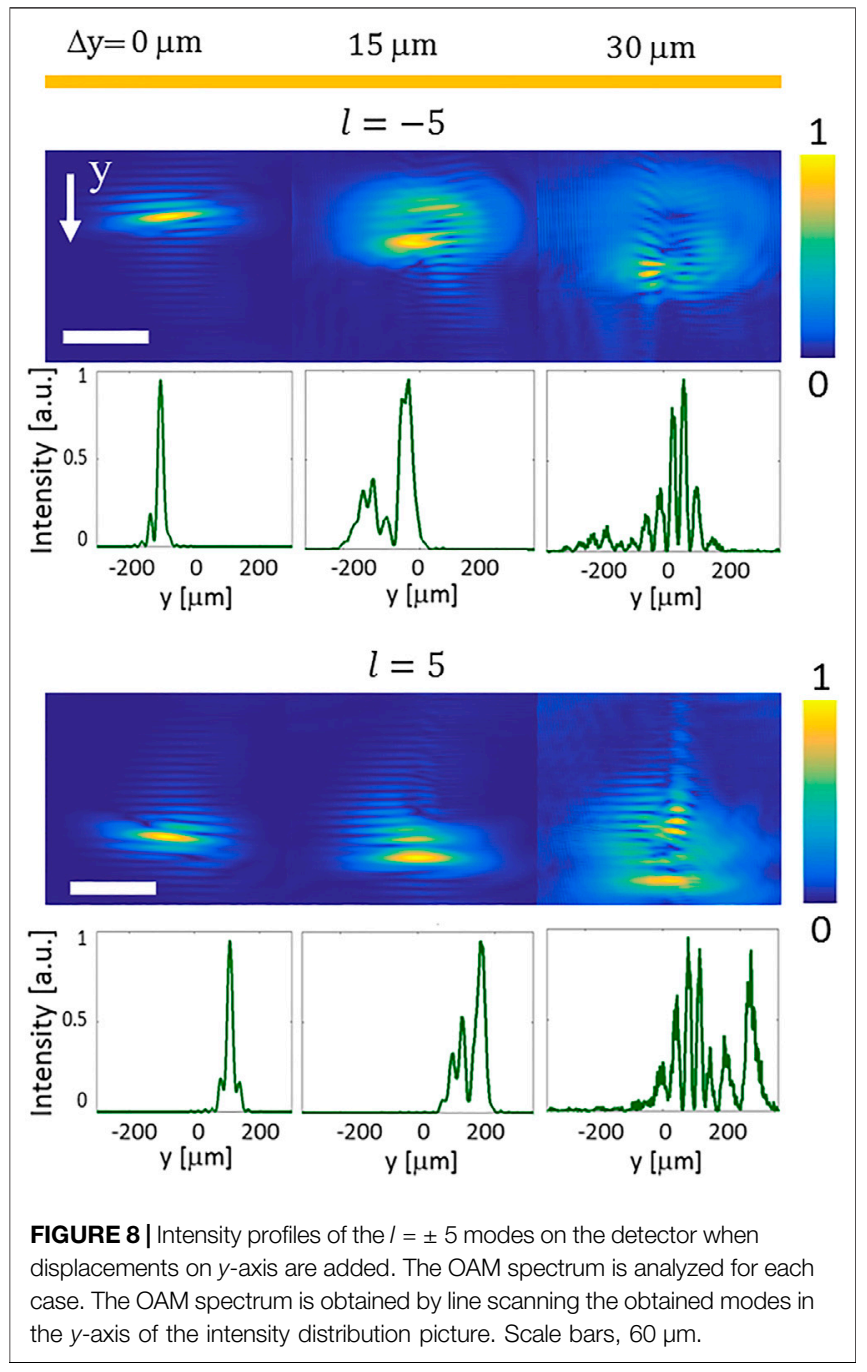

redistributed to regions $(a, b, c)$. Analysis of only partial areas of the phase distribution will lead to mixed states rather than a pure OAM determination. It can be seen that for different, smaller, and displaced regions, the OAM spectrum is increased $(\mathrm{a}, \mathrm{b})$, compared to a pure state (c). Figure 7 describes how the simulated beams appear on the detector. Here, the system was introduced to $l=0, \pm 2, \pm 4$ OAM states when also subjected to lateral displacements $(x=0,15 \mu m ; y=0,15 \mu m)$. The top row of Figure 7 describes a process without displacement, as center and bottom rows provide shifts of $15 \mu \mathrm{m}$ in $y$ - and $x$-axis, accordingly. Movement in the $y$-axis blurs the center spot, by broadening the lobes. Displacement in the orthogonal dimension narrows the lobes, as more fringes appear. Figure 8 provides spectrum analysis of $l= \pm 5$ states, for $\Delta y=$ $0,15,30 \mu \mathrm{m}$ displacements. The focused spots are accompanied with line scans ( $y$-axis) that provide the spectral broadening. It seems that there are no symmetrical effects for $l= \pm 5$. As expected, increasing the displacement up to $30 \mu \mathrm{m}$ decreases the modal purity, as the intensity of the peaks is rather similar. Meaning, it would be difficult to differentiate algorithmically the desired mode from the spectrum. To summarize these results, a light beam that has a partial vortex phase, or only partially detected, would induce wider

\section{$(x, y)$ :}
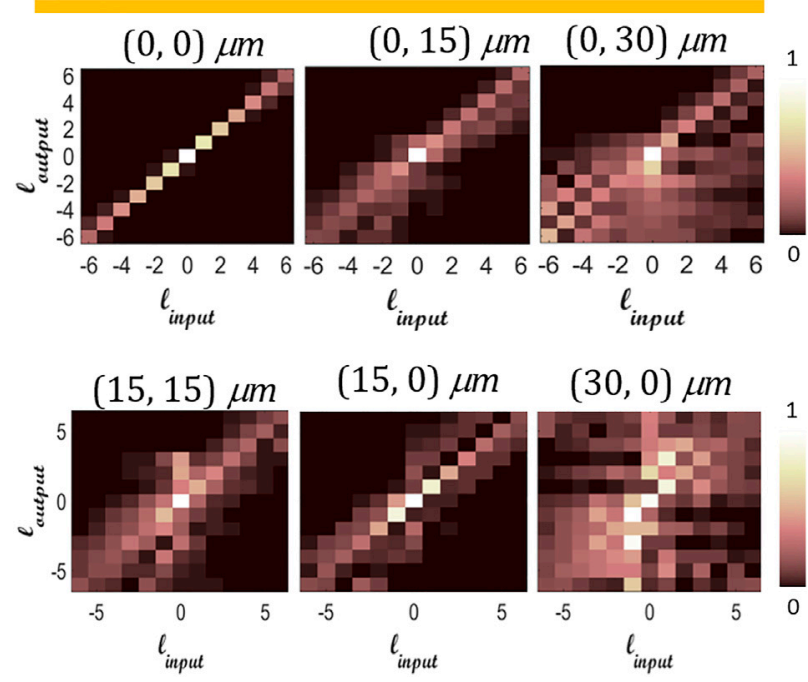

$(15,0) \mu m$

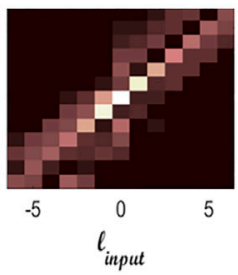

$(30,0) \mu m$

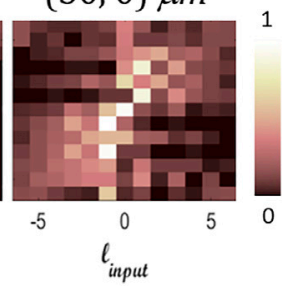

FIGURE 9 | Calculated cross-talk matrix for 13 modes, for various cases of system displacements $(15,30 \mu \mathrm{m})$.

OAM spectrum that interfere with the desired state sent. This is without even using any sorting devices. Since the mentioned modesorter is highly sensitive to misalignments, separating higher modes becomes challenging. This is manifested in wider OAM spectrum and additional fringes that screen the required specific state. It seems that small displacements, even as $10 \%$ from the sorter dimensions, induce sufficient disturbance to analyze the OAM states.

\section{THE SYSTEM'S MODAL CROSS-TALK}

Here, the cross-talk between various OAM states due to lateral and angular misalignment is investigated. This provides a two dimensional map of all modes there were sent and detected.

Another representation of the modal purity could be described as the modal cross-talk. In this approach, the system misalignment $(x, y \leq 30 \mu m)$ is analyzed, where the lateral size of each mode is plotted with respect to the modes that were sent versus those that were detected. By sending an OAM mode through the system and calculating the intensities in each subsection, it is possible to construct a $7 \times 7$ discrete cross-talk matrix for detecting all OAM modes, with respect to the existence of additional signal. Figure 9 presents the cross-talk matrices for six cases of misalignments, for $l \leq|6|$. It seems that displacement in $y$ provides distinguishable spectrum for $15 \mu \mathrm{m}$, as only positive states could be detected when reaching $30 \mu \mathrm{m}$. There is a spectrum broadening for displacement in $x$, as could be seen in the case of $(30 \mu \mathrm{m}, 0)$ compared to $(0,30 \mu \mathrm{m})$. So far, the cross talk was investigated upon lateral misalignment. Now the mode-sorter performance is examined with correlation to the angle of the incoming modes reaching the system's center $(0,0)$. In Figure 10, the cross-talk matrix is constructed by various incoming beams, with directionalities that equal to phase tilts of 3-12 $\mathrm{mrad}$, at each axis. For a small tilt of $3 \mathrm{mrad}$, there is no clear symmetry in both 


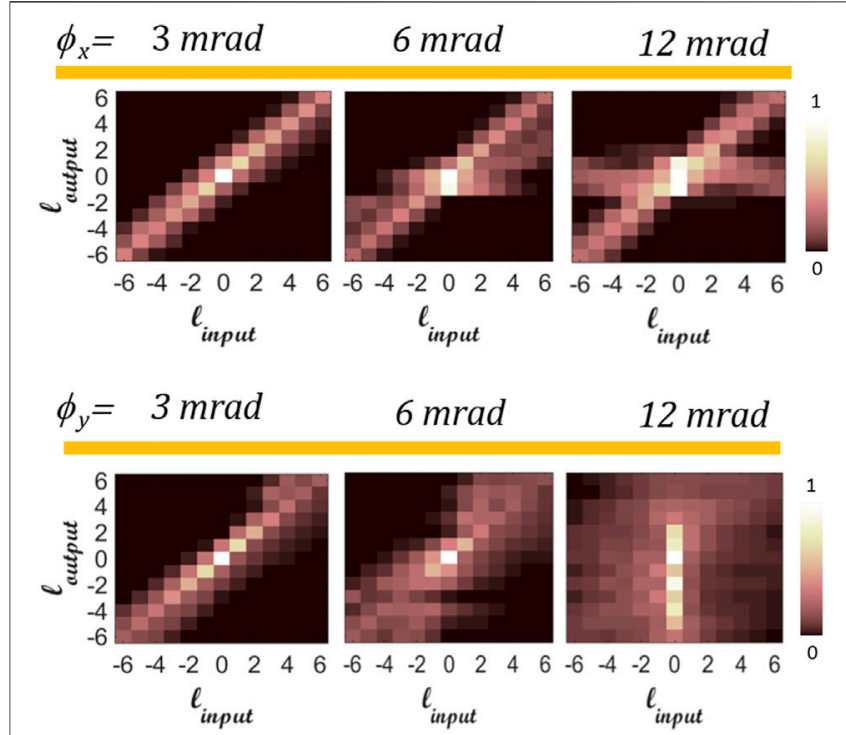

FIGURE 10 | Calculated cross-talk matrix for 13 modes, as the incoming modes propagate off-axis to the mode-sorter center. Each case describes modes that propagate with an additional linear phase gradient terms.

directions, and there is a spectral blur that exists in the $y$-axis. This effect further increases for stronger tilts in the $y$ direction. For the orthogonal $(x)$ direction, there is a rather unchanged spectrum width, as the tilt in $x$ increased. Furthermore, a strong blur effect disables the ability to analyze low-order modes. By analyzing the cross talk maps, it is clear to comprehend the necessary sensitivity requirements for such system. Small shifts and phase gradients damage the ability to analyze currently the OAM states. However, this sensitivity could be an advantage for secure communication systems as any interference could be noticed in the receiver plane. In any case, to eliminate their effects, the system may need to be designed differently, for example, printing the two elements as one for better accurate alignment, or by adding a light-collecting term that collects the shifted light to the elements, to reduce tilting issues.

\section{CONCLUSION}

The system is highly sensitive to misalignments, which is a disadvantage in most cases. However, this might be an advantage for private/secret communication, in which "listening" is almost impossible. The operation of the 3Dminiature mode-sorter system was examined. Two concerns were theoretically investigated, shedding more light on the system functionality to serve as a key element for future OAM-based communication systems: first, the system performance when misalignments (respect to the optical axis) are introduced; second, the ability of the mode-sorter to distinguish the OAM states as the beams arrive from various directions, as each beam has a different tilt, but still reach the center of the system. All calculations were based on a modesorter that was experimentally capable to separate pure and mixed OAM states, up to $l \leq|3|$; however, higher modes were also being investigated here. The cause for achieving limited experimental sorting capability $(l \leq|3|)$ is investigated as the vast calculation enabling shedding more light on this limitation. The calculative approach displaced the mode sorter laterally, in respect to the incoming beams as the performance was examined. Displacements of 15 and $30 \mu \mathrm{m}$ were chosen for $l \leq|5|$. From the results, the sorting process is sensitive to displacements of $15 \mu \mathrm{m}$ and above, as the ability to analyze the modes become challenging. Hence, a robust system should be printed directly on the optical axis of the entire system, which would limit misalignments to the printer precision lateral ability. The printed $3 \mathrm{D}$ is equipped with a $3 \mathrm{D}$-sensitive $(\mathrm{nm})$ piezo stage. Hence, a 3D-DLW process could be suitable for robust systems. The mode-sorter performance is sensitive to incoming beams, characterized with tilted phase fronts, except for values down to $3 \mathrm{mrad}$ which do not affect the system performance substantially. However, increasing the linear phase gradient to $12 \mathrm{mrad}$ would damage the ability to separate incoming OAM states. This effect is more dominant for one angle $\left(\varnothing_{y}\right)$, rather the perpendicular one $\left(\varnothing_{x}\right)$. For conclusion, the system can be operated for small OAM values, as the measurement system is well aligned. The power of the $3 \mathrm{D}$ DLW method allows allocating the printed elements with high precision according to the optical axis. This ability would improve the system demultiplexing performance.

The conclusion of the carried out analysis of the mode-sorter exhibits high sensitivity to optical and mechanical misalignments. This is commonly conceived as a disadvantage in most cases. However, this might be an advantage for secure communication, where the eavesdropper encounters signal broadening and impure states. Thus, our future goal is to construct a freespace integrated communication system, based on the reported insights. Furthermore, the mode-sorter could also be integrated on top of a suitable optical vortex fibers [26-28], combined with a collimating lens. This would enable precise alignment between the fiber core and the mode-sorter center, with low-probability for tilted modes, along with fiber-based communication system foothold.

\section{DATA AVAILABILITY STATEMENT}

The original contributions presented in the study are included in the article/Supplementary Material, further inquiries can be directed to the corresponding authors.

\section{AUTHOR CONTRIBUTIONS}

All authors listed have made a substantial, direct, and intellectual contribution to the work and approved it for publication.

\section{FUNDING}

This work was supported by the Pazy foundation. 


\section{REFERENCES}

1. Lightman S, Hurvitz G, Gvishi R, Arie A. Miniature Wide-Spectrum Mode Sorter for Vortex Beams Produced by 3D Laser Printing. Optica (2017) 4: 605-10. doi:10.1364/optica.4.000605

2. Allen L, Beijersbergen MW, Spreeuw RJC, Woerdman JP. Orbital Angular Momentum of Light and the Transformation of Laguerre-Gaussian Laser Modes. Phys Rev A (1992) 45:8185-9. doi:10.1103/physreva.45.8185

3. Garcés-Chávez V, McGloin D, Padgett MJ, Dultz W, Schmitzer H, Dholakia K. Observation of the Transfer of the Local Angular Momentum Density of a Multiringed Light Beam to an Optically Trapped Particle. Phys Rev Lett (2003) 91:93602. United States.

4. Hamazaki J, Morita R, Chujo K, Kobayashi Y, Tanda S, Omatsu T. Opticalvortex Laser Ablation. Opt Express (2010) 18:2144-51. doi:10.1364/oe.18. 002144

5. Swartzlander, Jr. GA, Ford EL, Abdul-Malik RS, Close LM, Peters MA, Palacios DM, et al. Astronomical Demonstration of an Optical Vortex Coronagraph. Opt Express (2008) 16:10200-7. OSA. doi:10.1364/oe.16.010200

6. García-Escartín JC, Chamorro-Posada P. Quantum Multiplexing with the Orbital Angular Momentum of Light. Phys Rev A - Mol Opt Phys (2008) 78:1-10.

7. Hell SW, Wichmann J. Breaking the Diffraction Resolution Limit by Stimulated Emission: Stimulated-Emission-Depletion Fluorescence Microscopy. Opt Lett (1994) 19:780-2. doi:10.1364/ol.19.000780

8. Bozinovic N, Yue Y, Ren Y, Tur M, Kristensen P, Huang H, et al. Terabit-Scale Orbital Angular Momentum Mode Division Multiplexing in Fibers. Science (2013) 340:1545-8.

9. Baghdady J, Miller K, Morgan K, Byrd M, Osler S, Ragusa R, et al. Multigigabit/s Underwater Optical Communication Link Using Orbital Angular Momentum Multiplexing. Opt Express (2016) 24:9794. doi:10.1364/oe.24. 009794

10. Lavery MPJ, Robertson DJ, Berkhout GCG, Love GD, Padgett MJ, Courtial J. Refractive Elements for the Measurement of the Orbital Angular Momentum of a Single Photon. Opt Express (2012) 20:2110-5. doi:10.1364/oe.20.002110

11. Berkhout GC, Lavery MP, Courtial J, Beijersbergen MW, Padgett MJ. Efficient Sorting of Orbital Angular Momentum States of Light. Phys Rev Lett (2010) 105:153601-11. doi:10.1103/PhysRevLett.105.153601

12. Ruffato G, Massari M, Parisi G, Romanato F. Test of Mode-Division Multiplexing and Demultiplexing in Free-Space with Diffractive Transformation Optics. Opt Express (2017) 25:7859. doi:10.1364/oe.25.007859

13. Ruffato G, Massari M, Romanato F. Compact Sorting of Optical Vortices by Means of Diffractive Transformation Optics. Opt Lett (2017) 42:551. doi:10. 1364/ol.42.000551

14. Mao D, Zheng Y, Zeng C, Lu H, Wang C, Zhang H, et al. Generation of Polarization and Phase Singular Beams in Fibers and Fiber Lasers. Adv Photon (2021) 3:1-18. doi:10.1117/1.ap.3.1.014002

15. Zhou X, Hou Y, Lin J. A Review on the Processing Accuracy of Two-Photon Polymerization. AIP Adv (2015) 5. doi:10.1063/1.4916886

16. Chichkov BN, Ostendorf A. Two-Photon Polymerization: A New Approach to Micromachining. Photon Spectra (2006) 40:72-9.
17. Schiappelli F, Kumar R, Prasciolu M, Cojoc D, Cabrini S, De Vittorio M, et al. Efficient Fiber-To-Waveguide Coupling by a Lens on the End of the Optical Fiber Fabricated by Focused Ion Beam Milling. Microelectronic Eng (2004) 7374:397-404. doi:10.1016/s0167-9317(04)00143-1

18. Kabouraki E, Giakoumaki AN, Danilevicius P, Gray D, Vamvakaki M, Farsari M. Redox Multiphoton Polymerization for 3D Nanofabrication. Nano Lett (2013) 13:3831-5. doi:10.1021/nl401853k

19. Thiel M, Fischer J, Von Freymann G, Wegener M. Direct Laser Writing of Three-Dimensional Submicron Structures Using a Continuous-Wave Laser at 532 Nm. Appl Phys Lett (2010) 97:10-3. doi:10.1063/1.3521464

20. Lightman S, Gvishi R, Hurvitz G, Arie A. Shaping of Light Beams by 3D Direct Laser Writing on Facets of Nonlinear Crystals. Opt Lett (2015) 40:4460. doi:10. 1364/ol.40.004460

21. Hossack WJ, Darling AM, Dahdouh A. Coordinate Transformations with Multiple Computer-Generated Optical Elements. J Mod Opt (1987) 34: 1235-50. doi:10.1080/09500348714551121

22. Bryngdahl O. Geometrical Transformations in Optics*. J Opt Soc Am (1974) 64:1092. doi:10.1364/josa.64.001092

23. Sinkin OV, Holzlohner R, Zweck J, Menyuk CR. Optimization of the Split-step Fourier Method in Modeling Optical-Fiber Communications Systems. J Lightwave Technol (2003) 21:61-8. OSA. doi:10.1109/jlt.2003.808628

24. Berry MV. Paraxial Beams of Spinning Light," in. Proc SPIE (1998) 3487.

25. Molina-Terriza G, Torres JP, Torner L. Management of the Angular Momentum of Light: Preparation of Photons in Multidimensional Vector States of Angular Momentum. Phys Rev Lett (2002) 88:4.

26. Jiang Y, Ren G, Shen Y, Xu Y, Jin W, Wu Y, et al. Two-dimensional Tunable Orbital Angular Momentum Generation Using a Vortex Fiber. Opt Lett (2017) 42:5014-7. doi:10.1364/ol.42.005014

27. Ramachandran S, Kristensen P. Optical Vortices in Fiber. Nanophotonics (2013) 2:455-74. doi:10.1515/nanoph-2013-0047

28. Brunet C, Vaity P, Messaddeq Y, LaRochelle S, Rusch LA. Design, Fabrication and Validation of an OAM Fiber Supporting 36 States. Opt Express (2014) 22: 26117. doi:10.1364/oe.22.026117

Conflict of Interest: The authors declare that the research was conducted in the absence of any commercial or financial relationships that could be construed as a potential conflict of interest.

Publisher's Note: All claims expressed in this article are solely those of the authors and do not necessarily represent those of their affiliated organizations, or those of the publisher, the editors, and the reviewers. Any product that may be evaluated in this article, or claim that may be made by its manufacturer, is not guaranteed or endorsed by the publisher.

Copyright (C) 2022 Lightman, Wengrowitcz, Teitelboim, Gvishi and Hurvitz. This is an open-access article distributed under the terms of the Creative Commons Attribution License (CC BY). The use, distribution or reproduction in other forums is permitted, provided the original author(s) and the copyright owner(s) are credited and that the original publication in this journal is cited, in accordance with accepted academic practice. No use, distribution or reproduction is permitted which does not comply with these terms. 